\title{
Robotic Lobectomy for Non-Small Cell Lung Cancer
}

\author{
Brian E. Louie
}

Received: 12 November 2012 / Accepted: 7 January 2013 /Published online: 12 February 2013

(C) Indian Association of Surgical Oncology 2013

\begin{abstract}
Over the last 5 years, there has been a tremendous increase in the interest in and use of robotics in thoracic surgery. The focus of this review is on the use of robotics for pulmonary lobectomy, which is being approached with 3 or 4 arm techniques. Early experiences suggest that the learning curve is approximately 20 cases for most surgeons but could be shortened with previous advanced thoracoscopic skills. Robotic lobectomy is feasible and safe in limited reports from experienced centers. Operative and clinical outcomes favor robotic lobectomy over open and appear to be similar to VATS. Limited data on oncologic effectiveness and survival suggest that robotics is similar to VATS. Widespread adoption and integration into practice will require future research efforts to prove oncologic and survival benefits in concert with cost effectiveness evaluation.
\end{abstract}

Keywords Robotics $\cdot$ Lung cancer $\cdot$ Lobectomy $\cdot$ VATS · Thoracotomy

Over the last 5 years, there has been a rapid evolution in minimally invasive surgery with increasing numbers of procedures across all specialties being transitioned from straight stick laparoscopy or thoracoscopy to the da Vinci surgical robot system with its 7 degrees of freedom endowrist instruments. This evolution, led by urology and gynecology, is now seeing the emergence of cardiothoracic surgery as the third largest surgical specialty adopting robotic technology [1]. The first reported thoracic procedures, as far back as 2004, were awkward, clumsy and took considerably more time but showed that robotic thoracic surgery including lobectomy was at least feasible [2,3]. At lot has changed since those first initial experiences by pioneering thoracic surgeons with pulmonary lobectomy, and segmentectomy,

\section{B. E. Louie $(\bowtie)$}

Minimally Invasive Thoracic Surgery Program, Swedish Cancer Institute and Medical Center, Suite 850, 1101 Madison Street, Seattle, WA 98104, USA

e-mail: brian.louie@swedish.org thymectomy, esophagectomy and mediastinal tumor resections all being regularly performed.

The purpose of this review is to review the history of video thoracic surgery (VATS), describe the technique of robotic lobectomy and its learning curve, review the current results of robotic lobectomy and highlight the potential future benefits and areas of investigation in robotic lung surgery.

\section{Past, Present and Future of Video Thoracoscopic Surgery}

Although simple thoracoscopy to examine the pleural space and perhaps perform simple wedge resection has existed for many decades, the advent of advanced thoracoscopic techniques applied to anatomic lung resection began in the early 1990's. Initial reports were not greeted with much enthusiasm but rather with skepticism and although the randomized trial by Kirby et al. [4] is heralded by many as a the start of an era of minimally invasive thoracic surgery, in retrospect it may have held back the development of VATS lobectomy because it told open surgeons that VATS did not confer any benefit over open muscle sparing thoracotomy. It's likely a generation of thoracic surgeons held back on becoming minimally invasive at a time when most other specialties were eager to embrace this new platform to perform surgery.

Now 17 years after that report, the number of lobectomies performed by VATS techniques has crept upward at a snails pace to now reach $32 \%$ [5] in the highly selective Society of Thoracic Surgeons database and a mere $6 \%$ in the Nationwide Inpatient Sample database [6]. However, in that time, research comparing VATS lobectomy to open lobectomy has shown the superiority of the minimally invasive approach in early stage lung cancers. Operative outcomes such as blood loss, operative times, peri operative complications all favor the minimally invasive approach [7]. Physiologically patients have improved post operative pulmonary function [8] and less inflammatory markers $[9,10]$. The oncologic benefits are similar to open approaches with similar 5 years survivals in 
early stage cancers [11] and similar lymph nodes staging [12]. In addition, the improved recovery facilitates the need to delivery adjuvant chemotherapy [13]. Patients are also seeing the benefits of less pain, reduced impact on pulmonary function, shorter length of stay, earlier return to usual activities and improved shoulder girdle function.

Despite all of the proven benefits, there remain concerns about the VATS approach being difficult to adopt $[14,15]$, the instability of the platform and in larger broader terms to show much difference outside of experienced centers. In addition, recent reviews have shown that over $70 \%$ of stage I lung cancers are performed open [16], the length of stay for VATS lobectomy in a recent query of the STS database was 5.3 days [17] and quite different than the 2 or 3 day length of stays being reported by experienced VATS surgeons [18] and that differences in nodal upstaging, when used as a surrogate for oncologic effectiveness in clinical stage I lung cancers, was minimized when high volume VATS surgeons were analyzed separately [16]. These findings support the notion that surgeons are still struggling with the VATS platform and the outcomes perhaps in the general thoracic community not equivalent to experienced VATS groups.

So what is the future of VATS lobectomy? Clearly further adoption and integration is necessary. However, it's likely that adoption of VATS techniques is going to plateau in the next several years as current trainees are all exposed. The limitations of this approach or perhaps the mindset of the surgeon will prevent it from reaching a greater rate of adoption. In order to increase the number of minimally invasive lung resections further, open surgeons will have to be convinced that robotic resection is feasible in their hands, reproducible, result in similar outcomes if not better than their current approach.

\section{Robotic Lung Resection}

Robotic lobectomy has garnered significant attention over the last 5 years with increasing numbers of surgeons becoming interested and an increasing number of cases being performed. Whether the attraction is purely one of marketing and technological interest or that surgeons believe that the stable platform, 10X magnification and binocular vision are the answer to the perceived limitations of VATS lobectomy is unclear. Fortunately, regardless of the surgeon's reasons, training and proctoring over the past 5 years have become more standardized. The main approaches to robotic lobectomy are described below.

\section{Patient Selection and Evaluation}

At the start of our robotic experience, patients were selected using similar criteria to patients offered VATS lobectomy.
Most of these were clinical stage I lung cancers that were peripherally located (outer third), less than $5 \mathrm{~cm}$ and suitable for lobectomy. Although patients were not selected based on the degree of emphysema, it is recommended to avoid patients with significant emphysema in the non-involved lobes until experience is gained with robotic techniques of moving the lung to avoid inadvertent trauma [19]. With increasing experience, these criteria have been extended to allow for larger, more central (middle third) tumors and those that require segmentectomy. Until more experience is gained, central tumors (inner third), T3 or T4 tumors, N3 disease, patients needing sleeve resection, and those having undergone induction therapy have been excluded. Patients have not been excluded based on BMI, ASA status, or age. In fact larger BMI, older patients and higher ASA may benefit from a minimally invasive approach rather than a thoracotomy.

Patients in our practice undergo standard radiologic staging with diagnostic $\mathrm{CT}$ of the chest to include in the adrenal glands, combined CT-PET imaging and selective MRI of the brain. Mediastinoscopy, when used is typically performed several days prior to robotic resection to maximize use of the robot. Although a tissue diagnosis not required before surgery, it is our preference to have a tissue diagnosis to preclude any need for a diagnostic wedge resection prior to lobectomy, which we find cumbersome on the robot. These two factors reduce the length of the procedure especially during the learning curve, facilitate the docking of the robot in an efficient manner, reduce concerns about local recurrence after wedge followed by completion lobectomy, and optimize appropriate staging before resection undertaken.

\section{Patient and Robotic Positioning}

Early on in robotic lobectomy there was no standard set up, port placement and instrument selection. Pioneering surgeons often adapted port placements from their VATS experience or from other surgeons until a set up was found to their liking. However, positioning and placement of the robotic arms often determines the ease of getting the case done. Two dominant approaches - one using 3 arms and one using 4 arms have emerged and been described [20,21].

Patients positioning is similar for virtually all open or VATS lobectomies with the patient in lateral decubitus. There is one exception; the operating room table is reversed to put the patient's head at the foot to allow for positioning of the robot (Fig. 1). Anesthesia is positioned to the face side of the patient to facilitate access to the double-lumen tube and the robot is positioned over the patient's head.

\section{Three Arm Set Up (CPRL-3)}

In a CPRL-3 robotic lobectomy, a total of 4 port are placed but only 2 robotic arms are used-numbers 1 and 3 with the 


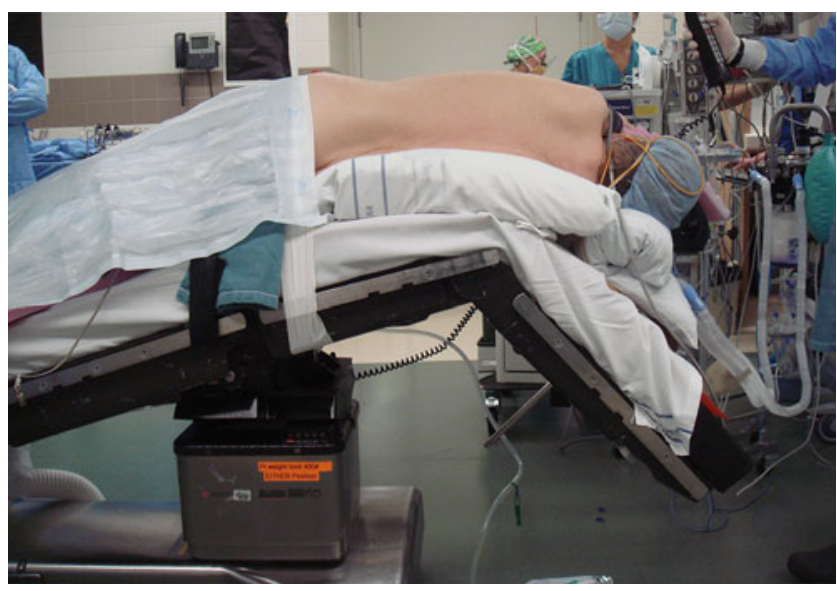

Fig. 1 Patient positioned on the operating table with the head of the patient on the foot end of the table

number 2 arms stored [21, 22]. The initial $8 \mathrm{~mm}$ port is placed in the sixth interspace anterior axillary line and is used with robotic arm 1 . Using the robotic $8-\mathrm{mm}$ camera, the remaining ports are placed under direct visualization based on internal anatomy and external position in the following order: $12-\mathrm{mm}$ camera port in the line of the scapula to enter the chest at the top of the diaphragm seen internally which usually coincides with the eight or ninth interspace and an 8-mm robotic arm 3 inferior and posterior to the scapular tip at the level of the superior segment. The fourth port was originally a 10 -mm port in the fourth interspace for suctioning or retraction but this is now an $11 \mathrm{~mm}$ laparoscopic port placed in the anterior axillary line to enter the chest just at the diaphragmatic insertion (Fig. 2). It is still used for retraction, stapling and suctioning by the bedside

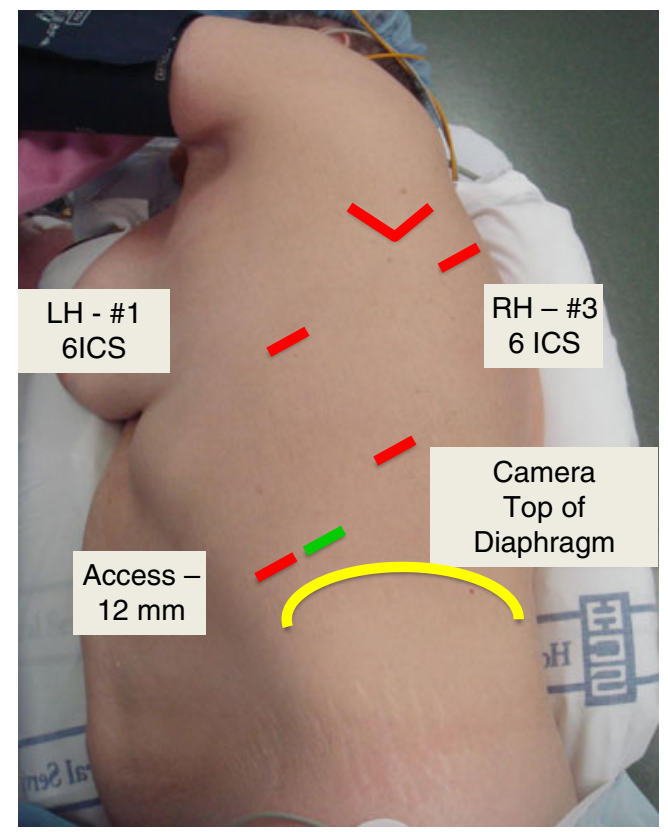

Fig. 2 Port placement for CPRL-3 assistant. Carbon dioxide insufflation is administered to a pressure of 6-8 $\mathrm{mmHg}$ with a flow of 6-8 $\mathrm{mL} / \mathrm{min}$ until the lung is deflated and then stopped. Four robotic instruments are used: Cadiere forceps, fenestrated bipolar, curved bipolar dissector, and L hook. Through the 11-mm port either a laparoscopic forceps is used retraction without grasping the lung or a suction-irrigation device is introduced. The specimen is placed in an extraction bag and removed by enlarging the camera port to approximately $5 \mathrm{~cm}$.

\section{Four Arm Set Up (CPRL-4)}

In a CPRL-4 robotic lobectomy, a total of 5 ports are placed but all 3 robotic arms are utilized (Fig. 3). The port placement for these is based on work by Cerfolio et al [20-23]. In this approach, the pleural space is entered with a $5-\mathrm{mm}$ port anteriorly in the midaxillary line at the top rib $7 . \mathrm{CO}_{2}$ insufflation is also used in this approach. A 5-mm VATS camera is inserted to placement of the remaining ports based on internal anatomy. The most posterior port $(5 \mathrm{~mm}, \mathrm{Arm} 3)$ is placed 2 rib spaces below the major fissure and just above the angle of the rib. Once this interspace is selected, all other ports are positioned in the same interspace moving from posterior to anterior as follows: Arm 2 is a $8 \mathrm{~mm}$ port placed $10 \mathrm{~cm}$ from the initial port; camera port is a 8 or $12 \mathrm{~mm}$ port placed $9 \mathrm{~cm}$ from Arm 2; Arm 1 is a $12 \mathrm{~mm}$ port placed $9 \mathrm{~cm}$ anterior to the camera port. A $15 \mathrm{~mm}$ access port is placed in the $9^{\text {th }}$ interspace mid to anterior axillary line. Stapling is performed via the $15 \mathrm{~mm}$ access port or by removing the instrument in Arm 1. The specimen is extracted by enlarging the $15 \mathrm{~mm}$ port to approximately $5 \mathrm{~cm}$.

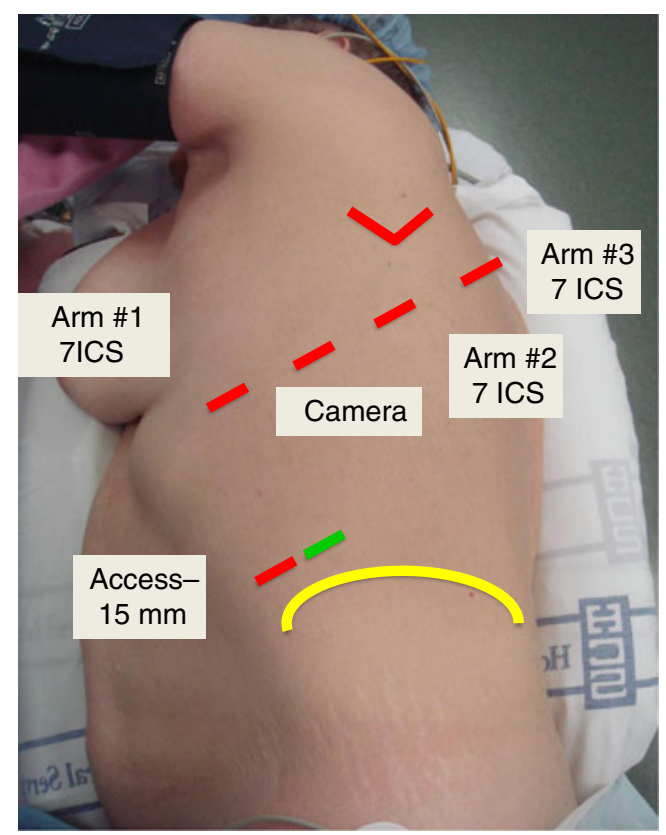

Fig. 3 Port placement for CPRL-4 


\section{Conduct of Robotic Lobectomy}

During the initial experience, it is advised to have two attending thoracic surgeons perform robotic lobectomy with one at the console and one at the bedside. This tends to maximize learning, patient safety and trouble shooting. My preference is to use a CPRL-3 set up since it is similar to the VATS lobectomy set up utilized before transition to the robot. This allows potential conversion to VATS if necessary and facilitates teaching residents and fellows who may not have access to a robot in practice.

The exact steps of the operation are surgeon and tumor dependent. In general, the non-involved lung is not grasped but pushed and manipulated by the flat surfaces of the robotic instruments - the so called "no-touch-technique". After division of the inferior pulmonary ligament and removal of station 9 lymph nodes, the inferior vein is exposed as necessary then the pleural reflection is divided posteriorly and the subcarinal space exposed and station 7 nodes removed. The posterior hilar structures are exposed such as the right station 11 node of Borrie or the left pulmonary artery and bronchus.

Attention is then turned to the anterior hilum where the pleura reflection is divided and the anterior hilum is exposed as necessary. For upper lobe cancers, we generally isolate and divide venous and arterial structures from anterior to posterior. For example, in a right upper lobectomy, the superior vein is divided followed by the pulmonary vessels and then the bronchus. The fissure is divided last. For lower lobe cancers, we generally move from inferior to superior dividing the inferior vein, bronchus and then pulmonary arteries. The fissure is divided last. Once the lobe is detached, it is placed inferiorly in the chest to facilitate further nodal assessment and resection.

Although this is a general approach, the exact steps will be dictated by the location and size of the tumor and the patient's anatomy. During hilar dissection, the operation is made easier by removal of the N1 nodes as they are encountered. This facilitates an understanding of the anatomy but also allows easy passage of surgical staplers.

\section{Learning Curve}

One of the current challenges for surgeons desirous of performing robotic lobectomy is mastering a new technology that is interposed between them and the patient. For all novice robotic surgeons, familiarity with the robotic system, patient positioning, docking the robot and knowing how to troubleshoot problems will be common learning experiences. However, transitioning a surgeon experienced in VATS lobectomy compared with a surgeon with limited or no experience in advanced thoracoscopic techniques may be different.

For experienced VATS surgeons, becoming facile on the robot should logically be simpler than an open surgeon with no VATS experience. When operative times and length of stay are used as a surrogate measure for the learning curve, it is possible to match historical VATS outcomes in as little as 6 cases [22]. However, in a different study comparing VATS and robotic lobectomy, operative times were significantly higher $($ robot $=240 \mathrm{~min}$ vs VATS $=161 \mathrm{~min}$ ) after the surgeon's first 40 robotic lobectomies compared to the most recent 40/148 VATS lobectomies [24]. These differences are potentially explained by the individual surgeon's volumes ( $>250 \mathrm{vs} 148$ ) and the presence of a single surgeon out of three performing robotic lobectomy compared to four surgeons all performing robotic lobectomy. In addition, the hospital experience with robotics in terms of docking, positioning and troubleshooting may have influenced operative times but this was not reported in either series.

The learning curve for surgeons with limited advanced thoracoscopic experience means that they have to learn a new set of techniques, be successful at the technique and integrate the technique. Veronesi et al. [25]. estimated the learning curve for a surgeon with no VATS experience at 18 robotic cases based on a statistically significant reduction in operative times comparing the first 18 and the next 73 cases. This is consistent with findings reported by others suggesting that robotic operative times will be 90 min longer than their colleagues with VATS experience [22] and other open surgeons transitioning to the robot [26].

At the current time, it appears that 20 cases is a reasonable learning curve for most thoracic surgeons. For surgeons with advanced thoracoscopic experience becoming comfortable with robotic lobectomy will likely require fewer cases and operative times will decrease quickly with increasing experience. It's also likely with a more standardized approach and consistent proctoring that the learning curve will be shorter for all surgeons compared to these early series when there was no standard port placement or conduct of the operation.

\section{Results of Robotic Lung Surgery}

Safety and Feasibility

A number of case series [3, 21, 26-28] and several comparative studies [20, 22, 24, 29] of robotic lung resection have now been published. It appears that anatomic lung resection, both lobectomy and recently segmentectomy [30], using a robotic platform is feasible and relatively safe. The inhospital or 30 day mortality rates range from 0 in most series and up to $4.9 \%$ in one series [19] although none of the deaths were due to the robotic technology but patient selection. These results are similar to VATS lobectomy and consistent with open lobectomy in the comparative series [20]. 
Conversion rates from robotic to open thoracotomy are higher than anticipated with some reports showing a nearly 1 in 5 conversion rate. However, one must remember that these reported outcomes likely represent the first robotic cases for all authors. In addition, several of the authors placed pre-defined time frames during their early robotic experiences for planned conversions to reduce surgeon and team frustration $[20,22]$. Most importantly, there were no conversions that resulted from bleeding and led to an operative death and most conversions were for adhesive disease, advanced T stage or lung isolation [27]. Until more experience and outcomes are reported from other academic and non-academic centers the feasibility and safety outcomes apply only to experienced centers.

\section{Morbidity}

Overall morbidity from robotic lobectomy ranges from 10 to $39 \%$ but major morbidity as high as $17 \%$ in one series, but this was similar to a match series of VATS lobectomies [22]. The most common morbidities were atrial fibrillation, prolonged air leak, pneumonia and respiratory distress. When compared to open thoracotomy robotic resection resulted in less morbidity [20,29] but when compared to VATS lobectomy was similar $[22,24]$. Recently presented studies using the Society of Thoracic Surgeons database [17] and the Nationwide Inpatient Sample and the State Inpatient Database [31] suggest that when robotic lobectomy is compared to larger populations of patients treated by general thoracic surgeons or by more generalist surgeons, the results still tend to favor the robot.

\section{Operative Outcomes}

There is little comparative data where the outcomes of robotic lobectomy are directly compared to standard VATS or open lobectomy. Logic dictates that robotic lobectomy will be superior to open thoracotomy in terms of operative and clinical outcomes such as length of stay and blood loss, very much like VATS is superior to open surgery these same parameters. In a recent systematic review, a meta-analysis comparing robot to open resection showed only a trend toward better peri-operative outcomes with robotic resection although length of stay was significantly shorter in the robotic cases [32]. Whereas, when robotic resection is compared to VATS there is no difference in blood loss, operative times or length of stay $[22,24]$.

\section{Patient Centered Outcomes}

Duration of pain or need for analgesia was reviewed in two series: one comparing to open thoracotomy [20] and one to VATS [22]. Both studies showed benefit from robotic resection in a shorter duration of pain or need for analgesia and the one study showed a faster return to usual activities. The rationale for a shorter duration of pain in the robotic group compared to open thoracotomy is expected but it is unclear whether the approach is superior to VATS without more data. It is possible the endowrist movements limit the levering of the instruments against the intercostal nerve, which is protective compared to the VATS approach where the straight stick instruments often press against the undersurface of the rib. One additional alternative explanation is that the repeated trauma from VATS instruments entering and exiting the chest may provide more pleural irritation leading to more pain. Until further, properly conducted studies using well executed pain assessments are completed, the advantage seems to favor robot over open thoracotomy.

One study assessed the quality of life in patients undergoing robotic lobectomy at 3 weeks and 16 weeks post operatively using the SF-12 [20]. At 3 weeks, patients undergoing robotic resection showed a significantly higher mental quality of life compared to open, rib-sparing thoracotomy, but the physical index only showed a trend toward favoring robotics. By the time patients were seen 16 weeks after surgery there were no differences. These findings seem consistent with the reduced analgesic requirements and earlier return to usual activities and suggest patients undergoing robotic lobectomy may have an earlier recovery but by 3 to 4 months there is no discernible difference.

\section{Oncologic Effectiveness and Survival}

There is little data to interpret whether robotic resection is oncologically effective since even the earliest studies are only now having patients reach the 5 year mark. Park et al. [27] reported an overall 5 year survival of $80 \%$ with median follow up of 27 months. This study included 325 consecutive patients with early clinical stage NSCLC from 3 institutions. By pathologic stage, the 5-year survival was similar to the most recent large series of VATS lobectomies.

Adoption and integration of robotic lobectomy into practice; however, is going to have to depend upon more than similar operative outcomes in the era of cost constraint. Robotic lobectomy is going to have show a survival or oncologic benefit. While survival data matures, one potential method to evaluate oncologic and surgical effectiveness would be to measure the rate of nodal upstaging from clinical staging to pathologic staging. Using the data from Park et al. [27], there appears to be some value in robotic lobectomy since upstaging of clinical stage I cancers may be higher $(21 \%)$ with robotic surgery when compared a recently reported STS database evaluation of over 11,500 lobectomies for clinical stage 1 cancers which yielded pathologic upstaging in the VATS group of $11.6 \%$ and in the open group of $14.3 \%$ [16]. 


\section{Cost of Robotic Surgery}

The apparent cost of robotic surgery is potentially its biggest challenge and ultimately may be the key breaking point for robotic surgery since the institution has to have the funds to purchase and then operate the system. Not surprisingly, the United States leads all countries in terms of purchased and installed robotic surgery systems whereas Canada, Europe and Asia whose health systems are more centralized have fewer. Nary a robot is seen in the developing world [1].

Even with purchased and operational systems, cost and cost-effectiveness are up front and center in most administrator's or government's minds. The only cost analysis published was performed using only 12 robotic cases and certainly does not reflect the current environment [33]. The results in this study showed that VATS lobectomy resulted in significantly less "cost" relative to open thoracotomy and placed the cost of robotics in between. The authors concluded that the reduction in length of stay was responsible for the difference between VATS and open lobectomy. It would seem that for robotic surgery to be cost effective it would have to achieve a shorter length of stay than VATS as well as showing more benefit.

The challenge in any study around cost will be the definitions of "cost" since there is no consistent methodology and how the capital and maintenance costs of the robot is amortized and accounted. In addition, the perspective taken during the analysis will also influence its conclusion since the interests of hospitals, insurers and society are selfishly different. Cost effectiveness will be best evaluated as part of a randomized trial comparing robotic lobectomy to VATS and open so that clinical outcomes and cost data are collected and analyzed prospectively, although I suspect this is unlikely to happen.

\section{Future Directions}

The future of robotic lobectomy appears to be bright and promising with early experiences with less than 50 cases having very similar outcomes compared mature VATS lobectomy results and improved outcomes when compared to open lobectomy. Patients have experienced an improvement in quality of life with reduced analgesia requirements and an early return work. Survival by stage is similar when compared to historically reported outcomes.

The true value of robotic lobectomy, given the early results, is not to convince experienced VATS surgeons to move on to the robot but to convince surgeon's who still favor open lobectomy for early stage NSCLC lung cancer. There remains considerable opportunity to increase the rate of minimally invasive lobectomy since the major of early stage lung cancers are still being resected with open thoracotomy.
Wide spread adoption of robotic surgery is likely 5 years away since the learning curve remains relatively steep especially for surgeons with limited advanced thoracoscopic skills. Trainees and experienced surgeons are encouraged to develop advanced thoracoscopic skills in the interim since it appears that these skills are helpful in the acquisition of robotic skills. In addition, while standardized port placement and operative techniques are emerging, there remains considerable work to make adoption and integration into one's practice easier. Newly trained robotic surgeons are encouraged to track their results and report them so that more can be learned about techniques and outcomes of robotic lobectomy. Lastly, robotic research focusing on the key issues of oncologic effectiveness and cost effectiveness is required to justify the initial investment and ongoing cost of maintenance. Several studies are underway to address these issues at several of these centers.

Disclosure The author discloses that he is on the speaker's bureau and a surgical proctor for Intuitive Surgical.

\section{References}

1. Investor Presentation Q3 2012 [Internet]. Intuitive Surgical. [cited 2012 Oct 10];Available from: http://phx.corporate-ir.net/phoenix. zhtml?c=122359\&p=irol-IRHome

2. Bodner J, Wykypiel H, Wetscher G, Schmid T (2004) First experiences with the da Vinci operating robot in thoracic surgery. Eur J Cardio Thorac Surg 25(5):844-851

3. Park BJ, Flores RM, Rusch VW (2006) Robotic assistance for video-assisted thoracic surgical lobectomy: technique and initial results. J Thorac Cardiovasc Surg 131(1):54-59

4. Kirby TJ, Mack MJ, Landreneau RJ, Rice TW (1995) Lobectomyvideo-assisted thoracic surgery versus muscle-sparing thoracotomy. A randomized trial. J Thorac Cardiovasc Surg 109(5):997-1001, discussion 1001-2

5. Boffa DJ, Allen MS, Grab JD, Gaissert HA, Harpole DH, Wright CD (2008) Data from the society of thoracic surgeons general thoracic surgery database: the surgical management of primary lung tumors. J Thorac Cardiovasc Surg 135(2):247-254

6. Gopaldas RR, Bakaeen FG, Dao TK, Walsh GL, Swisher SG, Chu D (2010) Video-assisted thoracoscopic versus open thoracotomy lobectomy in a cohort of 13,619 patients. Ann Thorac Surg 89(5): $1563-1570$

7. Paul S, Altorki NK, Sheng S, Lee PC, Harpole DH, Onaitis MW et al (2010) Thoracoscopic lobectomy is associated with lower morbidity than open lobectomy: a propensity-matched analysis from the STS database. J Thorac Cardiovasc Surg 139(2):366-378

8. Nakata M, Saeki H, Yokoyama N, Kurita A, Takiyama W, Takashima S (2000) Pulmonary function after lobectomy: videoassisted thoracic surgery versus thoracotomy. Ann Thorac Surg 70(3):938-941

9. Whitson BA, D'Cunha J, Andrade RS, Kelly RF, Groth SS, Wu B et al (2008) Thoracoscopic versus thoracotomy approaches to lobectomy: differential impairment of cellular immunity. Ann Thorac Surg 86(6): 1735-1744

10. Yim AP, Wan S, Lee TW, Arifi AA (2000) VATS lobectomy reduces cytokine responses compared with conventional surgery. Ann Thorac Surg 70(1):243-247 
11. Yan TD, Black D, Bannon PG, McCaughan BC (2009) Systematic review and meta-analysis of randomized and nonrandomized trials on safety and efficacy of video-assisted thoracic surgery lobectomy for early-stage non-small-cell lung cancer. J Clin Oncol 27(15):2553-2562

12. D'Amico TA, Niland J, Mamet R, Zornosa C, Dexter EU, Onaitis MW (2011) Efficacy of mediastinal lymph node dissection during lobectomy for lung cancer by thoracoscopy and thoracotomy. Ann Thorac Surg 92(1):226-231, discussion 231-2

13. Lee JG, Cho BC, Bae MK, Lee CY, Park IK, Kim DJ et al (2011) Thoracoscopic lobectomy is associated with superior compliance with adjuvant chemotherapy in lung cancer. Ann Thorac Surg 91(2):344-348

14. Yim APC (2010) Video-assisted thoracic lung surgery: is there a barrier to widespread adoption? Ann Thorac Surg 89(6):S2112-S2113

15. Rocco G, Internullo E, Cassivi SD, Van Raemdonck D, Ferguson MK (2008) The variability of practice in minimally invasive thoracic surgery for pulmonary resections. Thorac Surg Clin 18(3):235-247

16. Boffa DJ, Kosinski AS, Paul S, Mitchell JD, Onaitis M (2012 Jun 26) Lymph Node Evaluation by Open or Video-Assisted Approaches in 11,500 Anatomic Lung Cancer Resections. The Annals of Thoracic Surgery

17. Farivar AS, Cerfolio RJ, Vallières E, Knight A, Bryant A, Lingala $\mathrm{V}$, et al (Manuscript submitted). A Comparison of Robotic Anatomic Resection with Thoracotomy and VATS Resections Entered into the Society of Thoracic Surgery (STS) Registry. Innovations

18. McKenna RJ, Houck W, Fuller CB (2006) Video-assisted thoracic surgery lobectomy: experience with 1,100 cases. Ann Thorac Surg 81(2):421-425, discussion 425-6

19. Gharagozloo F, Margolis M, Tempesta B (2008) Robot-assisted thoracoscopic lobectomy for early-stage lung cancer. Ann Thorac Surg 85(6):1880-1885, discussion 1885-6

20. Cerfolio RJ, Bryant AS, Skylizard L, Minnich DJ (2011) Initial consecutive experience of completely portal robotic pulmonary resection with 4 arms. J Thorac Cardiovasc Surg 142(4):740-746

21. Dylewski MR, Ohaeto AC, Pereira JF (2011) Pulmonary resection using a total endoscopic robotic video-assisted approach. Semin Thorac Cardiovasc Surg 23(1):36-42

22. Louie BE, Farivar AS, Aye RW, Vallières E (2012) Early experience with robotic lung resection results in similar operative outcomes and morbidity when compared with matched videoassisted thoracoscopic surgery cases. Ann Thorac Surg 93(5): 1598-1605

23. Cerfolio RJ, Bryant AS, Minnich DJ (2011) Starting a robotic program in general thoracic surgery: why, how, and lessons learned. Ann Thorac Surg 91(6):1729-1736, discussion 1736-7

24. Jang H-J, Lee H-S, Park SY, Zo JI (2011) Comparison of the early robot-assisted lobectomy experience to video-assisted thoracic surgery lobectomy for lung cancer. Innovat (Philadelphia, PA) 6(5):305-310

25. Veronesi G, Agoglia BG, Melfi F, Maisonneuve P, Bertolotti R, Bianchi PP et al (2011) Experience with robotic lobectomy for lung cancer. Innovat (Philadelphia, PA) 6(6):355-360

26. Gharagozloo F, Margolis M, Tempesta B, Strother E, Najam F (2009) Robot-assisted lobectomy for early-stage lung cancer: report of 100 consecutive cases. Ann Thorac Surg 88(2):380-384

27. Park BJ, Melfi F, Mussi A, Maisonneuve P, Spaggiari L, Da Silva RKC et al (2012) Robotic lobectomy for non-small cell lung cancer (NSCLC): long-term oncologic results. J Thorac Cardiovasc Surg 143(2):383-389

28. Melfi F, Menconi GF, Mariani a M, Angeletti CA (2002) Early experience with robotic technology for thoracoscopic surgery. Eur J Cardio Thorac Surg 21(5):864-868

29. Veronesi G, Galetta D, Maisonneuve P, Melfi F, Schmid RA, Borri A et al (2010) Four-arm robotic lobectomy for the treatment of early-stage lung cancer. J Thorac Cardiovasc Surg 140(1):19-25

30. Pardolesi A, Park B, Petrella F, Borri A, Gasparri R, Veronesi G (2012) Robotic anatomic segmentectomy of the lung: technical aspects and initial results. Ann Thorac Surg 94(3):929-934

31. Kent MS, Wang T, Whyte R, Flores R, Gangadharan S. Open, VATS and Robotic Lobectomy: Review of National Database. Abstract: Southern Thoracic Surgical Association. Presented November 8, 2012

32. Cao C, Manganas C, Ang SC, Yan TD (2012) A systematic review and meta-analysis on pulmonary resections by robotic videoassisted thoracic surgery. Ann Cardiothorac Surg 1(1):3-10

33. Park BJ, Flores RM (2008) Cost comparison of robotic, videoassisted thoracic surgery and thoracotomy approaches to pulmonary lobectomy. Thorac Surg Clin 18:297-300 